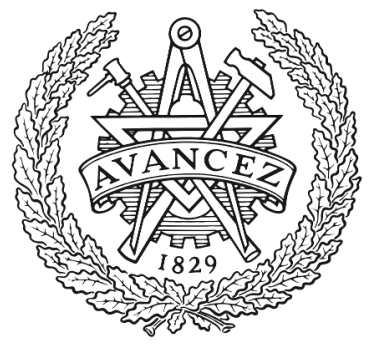

CHALMERS

UNIVERSITY OF TECHNOLOGY

\title{
Distributed Massive MIMO via all-Digital Radio Over Fiber
}

Downloaded from: https://research.chalmers.se, 2023-04-26 13:53 UTC

Citation for the original published paper (version of record):

Aabel, L., Durisi, G., Sezgin, I. et al (2020). Distributed Massive MIMO via all-Digital Radio Over Fiber. Conference Record - Asilomar Conference on Signals, Systems and Computers, 2020-November: 319-323. http://dx.doi.org/10.1109/IEEECONF51394.2020.9443347

N.B. When citing this work, cite the original published paper. 


\title{
Distributed Massive MIMO via all-Digital Radio Over Fiber
}

\author{
Lise Aabel $^{1,2}$, Giuseppe Durisi ${ }^{2}$, Ibrahim Can Sezgin ${ }^{2}$, Sven Jacobsson ${ }^{1}$, Christian Fager ${ }^{2}$, and Mikael Coldrey ${ }^{1}$ \\ ${ }^{1}$ Ericsson Research, Gothenburg, Sweden; e-mail: lise.aabel@ericsson.com \\ ${ }^{2}$ Chalmers University of Technology, Gothenburg, Sweden; e-mail: durisi@chalmers.se
}

\begin{abstract}
A crucial challenge in the implementation of distributed massive multiple-input multiple-output (MIMO) architectures is to provide phase coherence while, at the same time, limit the complexity of the remote-radio heads (RRHs), which is important for cost-efficient scalability. To address this challenge, we present in this paper a phase-coherent distributed MIMO architecture, based on off-the-shelf, low-cost components. In the proposed architecture, up- and down-conversion are carried out at the central unit $(\mathrm{CU})$. The RRHs are connected to the $\mathrm{CU}$ by means of optical fibers carrying oversampled radio-frequency (RF) 1-bit signals. In the downlink, the 1-bit signal is generated via sigma-delta modulation. At the RRH, the RF signal is recovered from the 1-bit signal through a bandpass filter and a power amplifier, and then fed to an antenna. In the uplink, the 1bit signal is generated by a comparator whose inputs are the lownoise-amplified received RF signal and a suitably designed dither signal. The performance of the proposed architecture is evaluated with satisfactory results both via simulation and measurements from a testbed.
\end{abstract}

\section{INTRODUCTION}

A distributed massive multiple-input multiple-output (MIMO) network consists of a large number of spatially distributed antennas connected to a central unit (CU), which serves simultaneously many user equipments on the same time-frequency resources [1]. Distributed massive MIMO combines the ability of massive MIMO architectures to precisely beamform the transmitted power at the desired locations in space [2], with increased robustness to shadow fading and spatially correlated channels [1].

One important challenge in distributed MIMO architectures is how to guarantee phase coherence between the remote radio heads (RRHs), which is crucial to achieve satisfactory performance, especially in the downlink [3]. In a conventional distributed MIMO implementation, baseband data is exchanged between the central unit (CU) and the RRHs via a fronthaul link, which limits fronthaul rates but requires that upand down-conversion is performed at the RRHs. To achieve phase coherence in such an architecture, one would need to keep synchronized the oscillators at the RRHs, which are needed for up- and down-conversion. This can be done by, for example, distributing a common clock to all the RRHs. Unfortunately, this solution is unfeasible, especially at the high

This work was partly supported by the Swedish Foundation for Strategic Research under grant ID19-0036, and by the Swedish Governmental Agency for Innovation Systems (VINNOVA) within the competence center ChaseOn. carrier frequencies that are relevant for mm-wave communications (although the distribution of a low-frequency common signal is possible, in centralized, tiled architectures [4]).

To address this challenge, we consider in this paper an architecture that relies on direct radio-frequency $(\mathrm{RF})$ sampling. Specifically, we present a time-division duplexing (TDD) alldigital radio-over-fiber $(\mathrm{RoF})$ distributed MIMO transceiver architecture, in which up- and down-conversion is performed in the digital domain at the $\mathrm{CU}$ to provide phase coherence, and a 1-bit quantized version of the RF digital signal is exchanged over fronthaul optical-fiber links connecting the $\mathrm{CU}$ to the RRHs. Performing up- and down-conversion at the $\mathrm{CU}$ results in a drastic simplification of the RRHs, which, in our architecture, consist simply of bandpass and lowpass filters, power amplifiers, low-noise amplifiers, variable gain amplifiers, comparators, antennas, and optical-to-electrical and electrical-to-optical converters. As we shall illustrate in this paper by means of simulations as well as measurements performed on a testbed, this approach results in a low-cost, lowcomplexity, scalable architecture, which provides satisfactory performance in terms of per-symbol mean square error (MSE).

\section{A. Previous Work}

All-digital RoF implementations of distributed MIMO have been considered previously in the literature. As far as the downlink is concerned, sigma-delta modulation has been successfully adopted to generate at the CU oversampled 1-bit digital RF signals which, after bandpass filtering at the RRHs, are delivered to the power amplifier and then transmitted over the air via the antenna [5]-[10].

The uplink is not as well investigated. An all-digital RoF single-antenna uplink was presented in [11], in which a subtractive dither signal is applied to the RF signal prior to 1-bit quantization to achieve satisfactory performance in terms of error-vector magnitude (EVM) of the demodulated symbols. Subtractive dithering involves subtracting the dither signal in the digital domain, which adds complexity to the architecture.

In [12], an all-digital RoF downlink using sigma-delta modulation and an uplink using nonsubtractive dithering and 1-bit quantization was studied, successfully showing that 1-bit digital RF signals can be used to connect the CU and the RRHs for both uplink and downlink, without the need of subtractive dithering. However, in the results reported in [12], the dither signal was produced using a dedicated waveform generator 


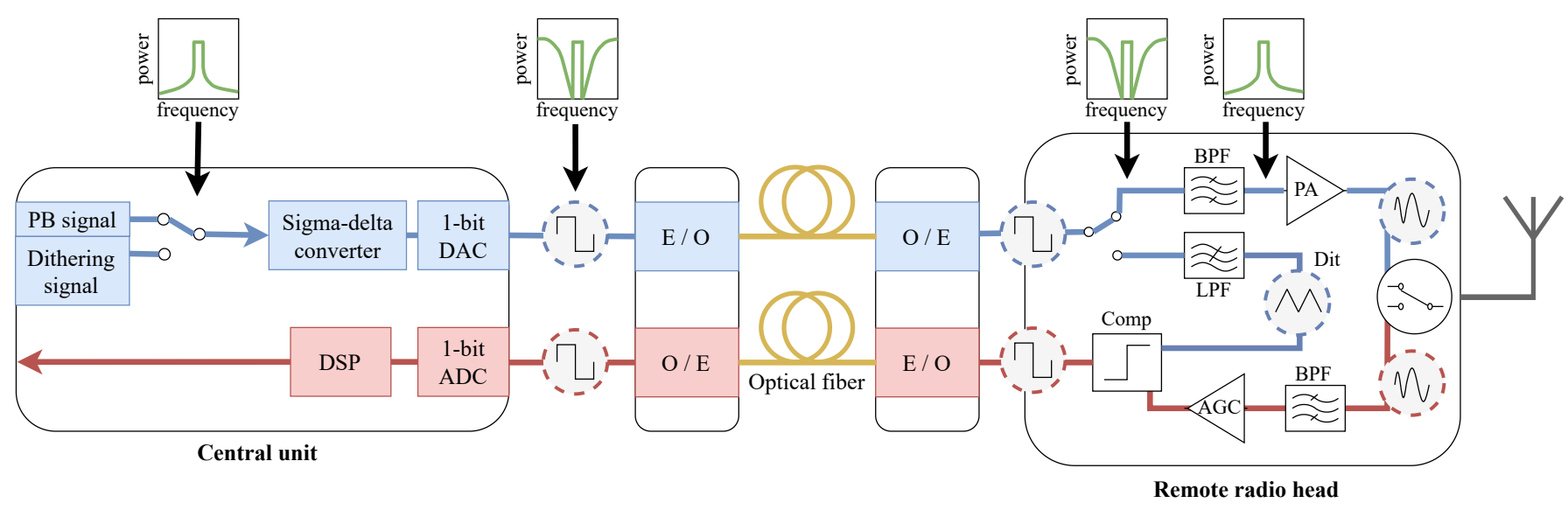

Fig. 1. The proposed transceiver architecture for the case in which the CU is connected to a single RRH. In downlink mode (blue traces and blocks), the digital pass-band (PB) signal is passed through a sigma-delta modulator, whose output is an oversampled 1-bit signal. After digital-to-analog conversion at the 1-bit DAC, the signal is converted to the optical domain ("E/O" and "O/E" in the figure stand for electrical-to-optical and optical-to-electrical conversion). At the RRH, the signal is converted to the electrical domain and then passed through a bandpass filter (BPF) and a power-amplifier (PA), and finally connected to the antenna. In uplink mode (red traces and blocks), the RF signal received by the antenna is passed through a low-noise amplifier (LNA), a BPF, an automatic gain control (AGC) and a comparator (Comp). In uplink mode, the transmitter provides a dither signal (Dit) to the comparator at the RRH, which is created at the CU, retrieved via a lowpass filter (LPF) at the RRH and fed to the comparator. The output of the comparator is converted to the optical domain and sent to the CU through the optical fiber. After 1-bit analog-to-digital conversion, this signal is finally sent to the digital signal processing (DSP) unit. To illustrate the effect of the various blocks on the PB signal in the downlink, we sketched in the figure its power spectrum (green plots) at various points in the transmitted chain as well as the corresponding time-domain signal (plots within the dashed circles).

located at the RRH, making the measurement setup stationary and prohibiting measurements involving several distributed links.

\section{B. Contributions}

Inspired by [12], we present a TDD all-digital RoF distributed MIMO architecture that involves low-cost components, and results in a scalable design. As in [12], the downlink relies on sigma-delta modulation and the uplink on 1-bit quantization of a dithered version of the RF signal. Differently from [12], the dither signal is generated at the $\mathrm{CU}$ and conveyed to the RRHs via the sigma-delta-over-fiber downlink. This enables the simultaneous distribution of the dither signal to multiple distributed RRHs. We study the performance of this architecture via simulations. Specifically, we show that a low per-symbol MSE can be achieved for system-parameter values of practical interest (16-QAM constellation, signal bandwidth of $10 \mathrm{MHz}$, carrier frequency of $2.4 \mathrm{GHz}, 10 \mathrm{GHz}$ sampling rate, for the case of a single RRH), despite the nonlinearity introduced by the 1-bit quantizers. Finally, we provide measurement results to show that the uplink structure proposed in the paper can be implemented with low-cost hardware components.

\section{All-Digital Transceiver Architecture}

\section{A. Uplink and Downlink Modes}

Our proposed transceiver architecture is depicted in Fig. 1 for the case when the $\mathrm{CU}$ is connected to one single-antenna RRH. The architecture can easily be expanded to multiple single-antenna RRHs. The main feature of the architecture is that the RRHs are connected via optical fibers to the
CU, where digital signal processing is performed, including digital up- and down-conversion. This guarantees phasecoherent transmission and reception. Processing RF signals in the digital domain requires high-speed digital-to-analog and analog-to-digital converters (DACs and ADCs). Since fast, high-precision ADCs are power hungry and difficult to implement, our solution involves the transmission of a 1-bit quantized version of the RF signal over the optical fiber. This all-digital RoF solution has the additional benefit of drastically lessening the linearity requirements on the optical fiber and can be implemented using low-cost commercial optical converters. This makes it more attractive than analog RoF solutions, which require high linearity. One disadvantage of our all-digital RoF solution is that, at least in the single RRH case, it requires one to significantly oversample the digital RF signal in order to obtain satisfactory performance in terms of, e.g., per-symbol MSE.

Referring to Fig. 1, we next provide some additional details on the transmitter architecture. In downlink mode, the digital pass-band signal is converted into a 1-bit digital signal by means of a sigma-delta modulator. This approach is chosen because it allows one to shape the spectrum of the quantization noise, so that it is mostly concentrated outside the bandwidth of the bandpass signal of interest. The signal at the output of the 1-bit DAC is converted into the optical domain (i.e., it is used to modulate a laser) and then transmitted over the optical fiber to the RRH. At the RRH, the signal is converted back to the electrical domain by means of a photo-detector. It is important to note that the use of 1-bit coding facilitates the use of standardized low-cost optical-communication components. The noise-shaping feature of the sigma-delta modulator allows us to recover the original pass-band signal by a simple 
bandpass-filtering operation. Finally, the resulting signal is amplified and transmitted via the antenna.

In uplink mode, the received signal at the antenna port of each RRH is amplified, filtered, and connected to one of the input ports of a comparator, whose purpose is to convert the RF signal into a binary signal. This is done by comparing the RF signal to a reference signal fed to the second input port of the comparator. The severe nonlinearity in the receiver chain introduced by the comparator results in a significant quantization error. To mitigate the impact of this quantization error, especially in the high-SNR regime, we apply nonsubtractive dithering to the RF signal-a common approach to improve the performance of low-precision quantizers by whitening the spectrum of the quantization noise.

To implement dithering, we connect a dither signal to one of the input ports of the comparator. The dither signal is provided to the RRH via the downlink chain. This is possible because our architecture operates in TTD mode. Hence, no downlink signal is transmitted over the downlink chain during the uplink phase. The dither signal is generated in the digital domain at the CU. Then, it is sigma-delta modulated, transmitted over the optical fiber, and reconstructed at the RRH via filtering. Similar to [12], the dither signal is chosen to be a triangular waveform, whose fundamental frequency is on the order of the bandwidth of the pass-band signal. Since this is a base-band signal, it can be recovered at the RRH using a lowpass filter. More details on the design of the dither signal are provided in Section II-C.

The two-level analog signal at the output port of the comparator is converted to the optical domain, transmitted over the optical fiber, converted back to the electrical domain, oversampled using a 1-bit ADC at the $\mathrm{CU}$, and then digitally processed.

\section{B. Downlink Sigma-Delta Conversion}

As pointed out in Section II-A, a key element of the transmitter architecture is the sigma-delta converter. Sigma-delta conversion provides a binary output signal, whose spectrum is shaped so that the quantization distortion that lies within the bandwidth of the input signal is minimized [13]. The noiseshaping properties of the sigma-delta converter are determined by two parameters: the oversampling ratio (OSR) and the order of the modulator. The OSR is defined as

$$
\text { OSR }=\frac{f_{s}}{2 B}
$$

where $f_{s}$ denotes the sampling frequency and $B$ is the bandwidth of the input signal. Since the sigma-delta modulator spreads the power of the quantization noise across the frequency interval $\left[0, f_{s}\right]$, and since the input signal has most of its power within a frequency interval of size $B$ centered at the carrier frequency $f_{c}$ satisfying $B \ll f_{c}<f_{s}$, the larger $f_{s}$, the lower the power of the in-band quantization error.

The order of the sigma-delta modulator determines the structure of the feedback loop used within this modulator (i.e., the number of integrators). The higher the order, the lower the quantization-noise power within the bandwidth of interest [13].

\section{Uplink Dithered 1-bit Quantization}

As mentioned in Section II-A, dithering the received signal before 1-bit quantization is crucial in our uplink architecture to achieve good performance at high-SNR values. As in [12], we use a triangular waveform as a dither signal, whichdifferently from [12]-is generated at the CU. Hence, its frequency and power can be controlled and optimized, so as to minimize, e.g., the per-symbol MSE. As we shall illustrate in Section IV, the optimal triangular dither signal has a frequency that is slightly larger than the bandwidth of the received signal. Furthermore, its power is on the same order of the power of the received signal after amplification. This means that an AGC needs to be used at the RRHs in uplink mode (see Fig. 1) to correctly scale up the RF signal so that its amplitude matches that of the chosen dither signal.

After sampling the RF signal at the CU, frequency downconversion is performed in digital domain through

$$
y_{\mathrm{BB}}[n]=y_{\mathrm{PB}}[n] e^{-j 2 \pi n f_{c} / f_{s}},
$$

where $y_{\mathrm{BB}}[n]$ is the down-converted baseband signal at discrete time $n$, and $y_{\mathrm{PB}}[n]$ is the corresponding received passband signal. Digital down-conversion is then followed by digital lowpass filtering, after which additional processing steps are applied to the uplink signal for demodulation and decoding.

\section{TESTBED}

At Chalmers University of Technology, an all-digital sigmadelta over-fiber downlink testbed has been implemented [6]see Fig. 2. All signal processing is performed offline in MATLAB, and the corresponding signals are loaded on a field-programmable gate array (FPGA) evaluation board. The selected FPGA evaluation board "Transceiver Signal Integrity Develeopment Kit, Stratix V GT Edition" can support a maximum of 14 digital transceiver links, operating at 12.5 $\mathrm{Gb} / \mathrm{s}$ each [14]. The optical converters used to transfer the digital RoF signals are small-form-factor, pluggable (SFP+) transceivers (AFBR-709SMZ, Avago Technologies), equipped with $850 \mathrm{~nm}$ vertical-cavity surface-emitting lasers, operating at $10 \mathrm{~Gb} / \mathrm{s}$. The $\mathrm{SFP}+$ optical transceivers provide differential input and output ports in electrical domain and binary optical transmission. Each RRH in Fig. 2 includes an SFP+, a bandpass filter, a power amplifier and an antenna.

We use the testbed described above also for performing uplink measurements. In uplink mode, the differential input ports of the SPF+ at the RRH are connected directly to the RF signal and the dither signal, since this component is designed to perform the comparator function (see Fig. 1). In order to implement all the required uplink operations, the RRH is equipped with an additional lowpass filter with a cut-off frequency of $200 \mathrm{MHz}$, which is used to recover the dither signal digitally generated at the CU. The optical fiber cables connecting the RRHs and the $\mathrm{CU}$ are $30 \mathrm{~m}$ long, multi-mode cables of type OM4. 


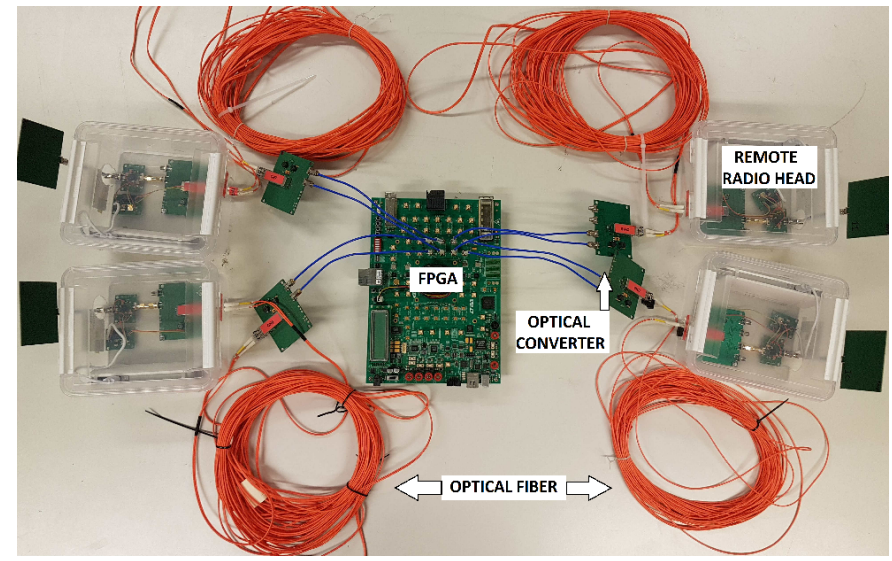

Fig. 2. Downlink testbed hardware: the figure shows the RRHs, the optical transceivers (SFP+), the optical-fiber cables, and the FPGA evaluation board.

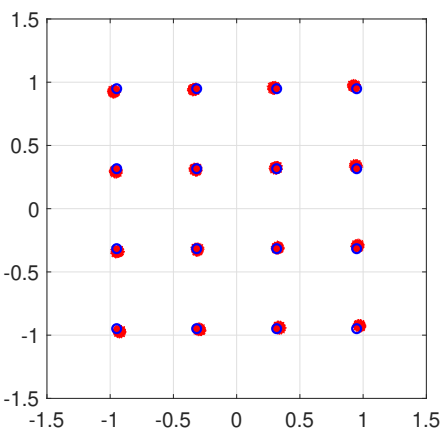

Fig. 3. Simulated 16-QAM symbols after sigma-delta conversion and bandpass filtering at the RRH. The signal bandwidth is $10 \mathrm{MHz}$, the carrier frequency is $2.4 \mathrm{GHz}$, the sample rate is $10 \mathrm{GHz}$ and the sigma-delta modulation order is four. The achieved per-symbol MSE is $-27 \mathrm{~dB}$.

\section{RESUlts}

\section{A. Downlink}

We present simulation results that illustrate the capability of sigma-delta conversion combined with bandpass filtering to overcome the nonlinearity introduced by the 1-bit DACs and provide good performance in terms of per-symbol MSE. We consider the case in which a single RRH is connected to the $\mathrm{CU}$. The pass-band signal is centered at $2.365 \mathrm{GHz}$, and contains 16-QAM symbols with $10 \mathrm{MHz}$ bandwidth. The sampling frequency $f_{s}$ used by the sigma-delta converter is $10 \mathrm{GHz}$, and its order is four. In the constellation diagram reported in Fig. 3, we plot the 16 QAM symbols after sigmadelta conversion and bandpass filtering of the RF signal at the RRH. The corresponding per-symbol MSE is $-32 \mathrm{~dB}$. This result shows that the in-band quantization distortion caused by the sigma-delta modulator is indeed small. Measurement results conducted on the downlink testbed, using the FPGA evaluation board, the SFP+ optical transceivers, the bandpass filter and the power amplifier, confirm that the impact of the in-band quantization noise generated in the sigma-delta conversion is marginal [6].
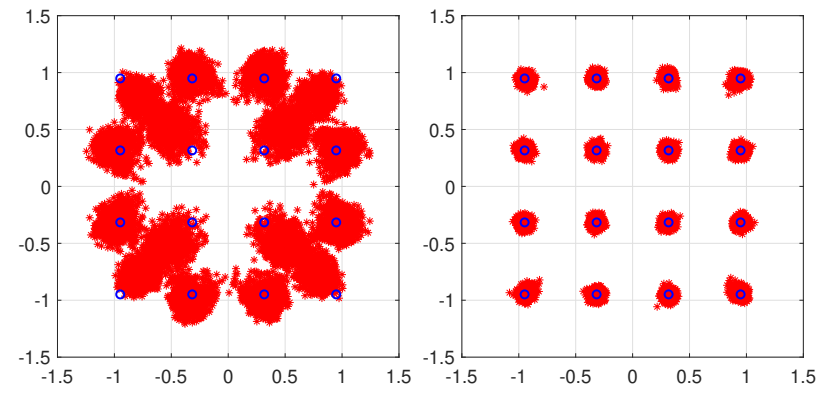

Fig. 4. Simulated constellation diagrams of received symbols after 1-bit quantization performed at $10 \mathrm{GHz}$, for the case of a single RRH. The transmitted RF signal has a central frequency of $2.365 \mathrm{GHz}$ and contains 16-QAM symbols with $10 \mathrm{MHz}$ bandwidth. This signal is passed through an AWGN channel with $10 \mathrm{~dB}$ SNR. Without dithering (left), the per-symbol MSE is $-13 \mathrm{~dB}$, whereas when dithering is used (right), the per-symbol MSE is $-27 \mathrm{~dB}$.

\section{B. Uplink}

To study the uplink performance via simulation, we assume that the transmitted RF signal is single-carrier modulated, centered at $2.365 \mathrm{GHz}$ and contains 16-QAM symbols with 10 $\mathrm{MHz}$ bandwidth. A root-raised cosine waveform with roll-off factor 0.2 is used for pulse shaping. The RF signal is passed through an AWGN channel with $10 \mathrm{~dB}$ SNR. The dither signal used at the RRH is a triangular waveform at $12 \mathrm{MHz}$ and the ratio between the dither-signal power and the RF-signal power is set to $2 \mathrm{~dB}$. In Fig. 4, we present the constellation diagram of the demodulated symbols at the CU. As shown in the figure, when no dithering is applied to the RF signal at the RRH, the per-symbol MSE is $-13 \mathrm{~dB}$. As expected, with dithering we can improve the per-symbol MSE to $-27 \mathrm{~dB}$.

In Fig. 5, we report measured per-symbol MSE values as a function of the ratio between the power of the dither signal and the power of the RF signal, measured at the input ports of the SFP+. In the measurement setup, an RF signal centered at $2.365 \mathrm{GHz}$ and containing 16-QAM symbols with $10 \mathrm{MHz}$ bandwidth is generated using the E4438C ESG Agilent vector signal generator. This RF signal is connected to one of the differential-input ports of the SFP+ optical transceiver. We then generate in MATLAB a triangular dither signal with a fundamental frequency of $20 \mathrm{MHz}$, pass it through a sigmadelta modulator, and upload it to the FPGA evaluation board. The dither signal is then transmitted over the optical fiber and is filtered at the RRH using a $200 \mathrm{MHz}$ lowpass filter. The cut-off frequency of the filter is chosen to balance the number of preserved frequency harmonics of the triangular waveform with the amount of in-band quantization noise after filtering. With our choice, the fundamental frequency as well as four harmonics of the triangular waveform are preserved. Finally, the dither signal is connected to the second differential-input port of the SFP+. The output of the comparator is transmitted over the optical fiber and then 1-bit sampled at $10 \mathrm{GHz}$ at the FPGA evaluation board. As shown in Fig. 5, for this measurement setup, the lowest MSE is achieved when the ratio between the dither-signal power and the RF-signal power is set 


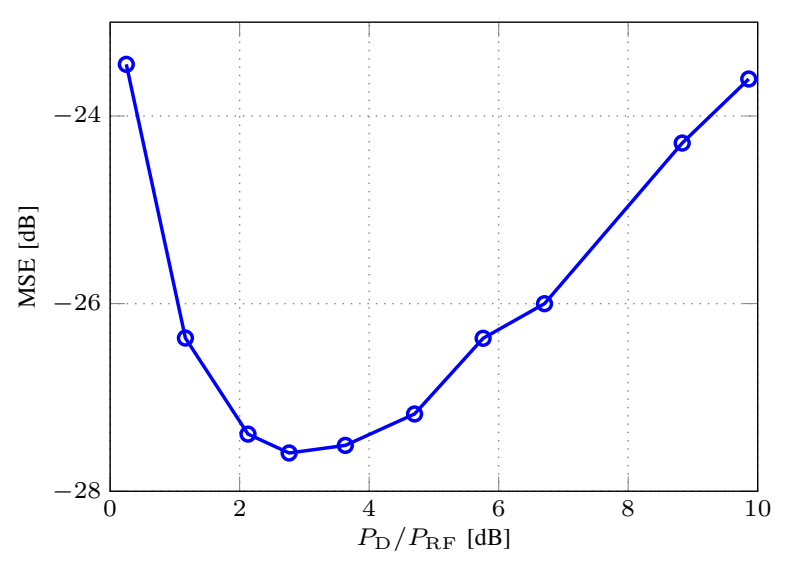

Fig. 5. Measured per-symbol MSE at the CU, as a function of the ratio between the power $P_{\mathrm{D}}$ of the dither signal and the power $P_{\mathrm{RF}}$ of the RF signal. The RF signal is centered at $2.365 \mathrm{GHz}$ and contains $64-\mathrm{QAM}$ symbols with $10 \mathrm{MHz}$ bandwidth. The sampling frequency at the $\mathrm{CU}$ is $10 \mathrm{GHz}$.

to $2.7 \mathrm{~dB}$. These results are in agreement with the simulation results reported in [15], for a slightly different scenario, which involves the transmission of orthogonal frequency-division multiplexing signals.

\section{CONClusion}

We have presented an all-digital radio-over-fiber architecture that provides phase-coherent links in a distributed massive MIMO deployment. The architecture is based on off-the-shelf, low-cost components. Hence, it is scalable.

As shown both via simulation results and measurements performed on a testbed developed at Chalmers University of Technology, the use of oversampling combined with sigmadelta modulation in the downlink and dithering in the uplink yield satisfactory performance, measured in terms of MSE of the estimated constellation symbols, despite the nonlinearity introduced by the 1-bit converters.

The results reported in this paper pertain to the scenario in which a single RRH is connected to the CU. Measurements involving multiple distributed RRHs will be presented in future publications, together with an analysis of the tradeoff between spatial oversampling obtained by using multiple RRHs and temporal oversampling obtained by increasing the OSR.

\section{REFERENCES}

[1] H. Q. Ngo, A. Ashikhmin, H. Yang, E. G. Larsson, and T. L. Marzetta, "Cell-free massive MIMO versus small cells," IEEE Trans. Commun., vol. 16, no. 3, pp. 1834-1850, Mar. 2017.

[2] T. L. Marzetta, E. G. Larsson, H. Yang, and H. Q. Ngo, Fundamentals of massive MIMO. Cambridge, U.K.: Cambridge Univ. Press, 2016.

[3] M. R. Khanzadi, G. Durisi, and T. Eriksson, "Capacity of SIMO and MISO phase-noise channels with common/separate oscillators," IEEE Trans. Commun., vol. 63, no. 9, pp. 3218-3231, Sep. 2015.

[4] M. E. Rasekh, M. Abdelghany, U. Madhow, and M. Rodwell, "Phase noise analysis for mmwave massive MIMO: a design framework for scaling via tiled architectures," in Proc. Conf. Infor. Sci. and Syst. (CISS), Baltimore, MD, USA, Mar. 2019.

[5] C. Y. Wu, H. Li, O. Caytan, J. Van Kerrebrouck, L. Breyne, J. Bauwelinck, P. Demeester, and G. Torfs, "Distributed multi-user MIMO transmission using real-time sigma-delta-over-fiber for next generation fronthaul interface," J. Lightw. Technol., vol. 38, no. 4, pp. 705-713, Feb. 2020.
[6] I. C. Sezgin, M. Dahlgren, T. Eriksson, M. Coldrey, C. Larsson, J. Gustavsson, and C. Fager, "A low-complexity distributed-MIMO testbed based on high-speed sigma-delta-over-fiber," IEEE Trans. Microw. Theory Techn., vol. 67, no. 7, pp. 2861-2872, Jul. 2019.

[7] I. C. Sezgin, T. Eriksson, J. Gustavsson, and C. Fager, "Evaluation of distributed MIMO communication using a low-complexity sigma-deltaover-fiber testbed," in IEEE MTTS Int. Microw. Symp., Boston, MA, USA, Jun. 2019, pp. 754-757.

[8] J. Wang, Z. Jia, L. A. Campos, and C. Knittle, "Delta-sigma modulation for next generation fronthaul interface," J. Lightw. Technol., vol. 37, no. 12, pp. 2838-2850, Jun. 2019.

[9] C. Y. Wu, H. Li, J. Van Kerrebrouck, A. Vandierendonck, I. L. De Paula, L. Breyne, O. Caytan, S. Lemey, H. Rogier, J. Bauwelinck, P. Demeester, and G. Torfs, "Distributed antenna system using sigmadelta intermediate-frequency-over-fiber for frequency bands above 24 GHz," J. Lightw. Technol., vol. 38, no. 10, pp. 2764-2772, May 2020.

[10] L. Bogaert, J. Van Kerrebrouck, L. Breyne, J. Lambrecht, H. Li, K. Van Gasse, J. Verbist, M. Vanhoecke, H. Ramon, S. A. Srinivasan, P. De Heyn, J. Van Campenhout, P. Ossieur, P. Demeester, X. Yin, J. Bauwelinck, G. Torfs, and G. Roelkens, "SiGe EAM-based transceivers for datacenter interconnects and radio over fiber," IEEE $J$. Sel. Topics Quantum Electron., vol. 27, no. 3, pp. 1-13, May-Jun 2021.

[11] A. Prata, A. S. Oliveira, and N. B. Carvalho, "Evaluation of distributed MIMO communication using a low-complexity sigma-delta-over-fiber testbed," in IEEE MTTS Int. Microw. Symp., San Francisco, CA, USA, May 2016, pp. 1-4.

[12] R. F. Cordeiro, A. Prata, A. S. Oliveira, J. M. Vieira, and N. B. De Carvalho, "Agile all-digital RF transceiver implemented in FPGA," IEEE Trans. Microw. Theory Techn., vol. 65, no. 11, pp. 4229-4240, Nov. 2017.

[13] S. Pavan, R. Schreier, and G. C. Temes, Understanding Delta-Sigma Data Converters, 2nd ed., R. Jacob Baker, Ed. Hoboken, NJ, USA: John Wiley \& Sons, 2017.

[14] Altera Corporation, "Transceiver signal integrity development kit, Stratix V GT edition reference manual," Jan. 2016.

[15] S. Jacobsson, L. Aabel, M. Coldrey, I. C. Sezgin, C. Fager, G. Durisi, and C. Studer, "Massive MU-MIMO-OFDM uplink with direct RFsampling and 1-bit ADCs," in Proc. IEEE Global Telecommun. Conf. (GLOBECOM), Waikoloa, HI, USA, Dec. 2019. 